\title{
Application of Rational Second Kind Chebyshev Functions for System of Integrodifferential Equations on Semi-Infinite Intervals
}

\author{
M. Tavassoli Kajani, ${ }^{1}$ S. Vahdati, ${ }^{2}$ Zulkifly Abbas, $^{3}$ \\ and Mohammad Maleki ${ }^{4}$ \\ ${ }^{1}$ Department of Mathematics, Islamic Azad University, Khorasgan Branch, 81515-158 Isfahan, Iran \\ ${ }^{2}$ Department of Mathematics, Khansar Faculty of Computer and Mathematics, \\ University of Isfahan, Isfahan 81746-73441, Iran \\ ${ }^{3}$ Department of Physics, Faculty of Science, University Putra Malaysia, 43400 Serdang, Selangor, Malaysia \\ ${ }^{4}$ Department of Mathematics, Islamic Azad University, Mobarakeh Branch, 84819-14411 Isfahan, Iran
}

Correspondence should be addressed to Zulkifly Abbas, za@science.upm.edu.my

Received 22 September 2012; Accepted 8 December 2012

Academic Editor: Mehmet Sezer

Copyright (C) 2012 M. Tavassoli Kajani et al. This is an open access article distributed under the Creative Commons Attribution License, which permits unrestricted use, distribution, and reproduction in any medium, provided the original work is properly cited.

Rational Chebyshev bases and Galerkin method are used to obtain the approximate solution of a system of high-order integro-differential equations on the interval $[0, \infty)$. This method is based on replacement of the unknown functions by their truncated series of rational Chebyshev expansion. Test examples are considered to show the high accuracy, simplicity, and efficiency of this method.

\section{Introduction}

In recent years, there has been a growing interest in the system of integrodifferential equations (IDE), which arise frequently in many applied areas which include engineering, mechanics, physics, chemistry, astronomy, biology, economics, potential theory, electrostatics, and so forth [1-8]. The systems of integrodifferential equations are generally difficult to solve analytically, thus finding efficient computational algorithms for obtaining numerical solution is required.

There are various techniques for solving systems of IDE, for example, operational Tau method [9, 10], Adomian decomposition method [11], Galerkin method [12], rationalized Haar functions method [13], He's homotopy perturbation method (HPM) [14, 15], and Ghebyshev polynomial [16].

A number of problems arising in science and engineering are set in semi-infinite domains. One can apply different spectral methods that are used to solve problems in 
semi-infinite domains. The first approach is using Laguerre polynomials [17-20]. The second approach is replacing semi-infinite domain with $[0, L]$ interval by choosing $L$, sufficiently large. This method is named domain truncation [21]. The third approach is reformulating original problem in semi-infinite domain to singular problem in bounded domain by variable transformation and then using the Jacobi polynomials to approximate the resulting singular problem [22]. The fourth approach of spectral method is based on rational orthogonal functions.

Boyd [23] defined a new spectral basis, named rational Chebyshev functions on the semi-infinite interval, by mapping to the Chebyshev polynomials. Guo et al. [24] introduced a new set of rational Legendre functions which are mutually orthogonal in $L^{2}(0,+\infty)$. They applied a spectral scheme using the rational Legendre functions for solving the Korteweg-de Vries equation on the half line. Boyd et al. [25] applied pseudospectral methods on a semiinfinite interval and compared rational Chebyshev, Laguerre, and mapped Fourier sine.

The authors of [26-29] applied spectral method to solve nonlinear ordinary differential equations on semi-infinite intervals. Their approach was based on a rational Tau method. They obtained the operational matrices of derivative and product of rational Chebyshev and Legendre functions and then they applied these matrices together with the Tau method to reduce the solution of these problems to the solution of system of algebraic equations.

Zarebnia and Ali Abadi [30] used Sinc-Collocation method for solving system of nonlinear second-order integrodifferential equations of the Fredholm type. Rational second (third) kind Chebyshev (RSC) functions, for the first time, were proposed by Tavassoli Kajani and Ghasemi Tabatabaei [31] to find the numerical solution of Lane-Emden equation.

This paper outlines the application of rational second kind Chebyshev functions and Galerkin method to the following system of linear high-order integrodifferential equations on the interval $[0, \infty)$. Two problems of such equations are solved to make clear the application of the proposed method. One has

$$
\begin{gathered}
\sum_{i=1}^{l}\left(\sum_{j=0}^{m} v_{p i j}(x) y_{i}^{(j)}(x)+\lambda_{i p} \int_{a}^{b}\left(k_{i p}(x, t) y_{i}(t)\right) d t\right)=f_{p}(x), \quad p=1,2, \ldots, l, \\
y_{i}^{(j)}(0)=y_{i j}, \quad i=1,2, \ldots, l, j=0,1, \ldots, m-1, \\
x \in[0, \infty),
\end{gathered}
$$

where $0 \leq a<b<\infty$.

\section{Properties of RSC Functions}

In this section, we present some properties of RSC functions.

\subsection{RSC Functions}

The second kind Chebyshev polynomials $U_{n}(x), n \geq 0$, are orthogonal in the interval $[-1,1]$ with respect to the weight function $\sqrt{1-x^{2}}$ and we find that $U_{n}(x)$ satisfies the recurrence relation [32]

$$
\begin{gathered}
U_{0}(x)=1, \quad U_{1}(x)=2 x \\
U_{n}(x)=2 x U_{n-1}(x)-U_{n-2}(x), \quad n \geq 2 .
\end{gathered}
$$


The RSC functions are defined by [31, 33]

$$
R_{n}(x)=U_{n}\left(\frac{x-1}{x+1}\right)
$$

thus RSC functions satisfy

$$
\begin{gathered}
R_{0}(x)=1, \quad R_{1}(x)=2\left(\frac{x-1}{x+1}\right) \\
R_{n}(x)=2\left(\frac{x-1}{x+1}\right) R_{n-1}(x)-R_{n-2}(x), \quad n \geq 2 .
\end{gathered}
$$

\subsection{Function Approximation}

Let $w(x)=4 \sqrt{x} /(x+1)^{3}$ denotes a nonnegative, integrable, real-valued function over the interval $I=[0,+\infty)$. We define

$$
L_{w}^{2}(I)=\left\{y: I \rightarrow \mathbb{R} \mid y \text { is measurable and }\|y\|_{w}<\infty\right\},
$$

where

$$
\|y\|_{w}=\left(\int_{0}^{\infty}|y(x)|^{2} w(x) d x\right)^{1 / 2}
$$

is the norm induced by the scalar product

$$
\langle y, z\rangle_{w}=\int_{0}^{\infty} y(x) z(x) w(x) d x
$$

Thus $\left\{R_{n}(x)\right\}_{n \geq 0}$ denote a system which is mutually orthogonal under (2.6), that is,

$$
\int_{0}^{\infty} R_{n}(x) R_{m}(x) w(x) d x=\frac{\pi}{2} \delta_{n m}
$$

where $\delta_{n m}$ is the Kronecker delta function. This system is complete in $L_{w}^{2}(I)$; as a result, any function $y \in L_{w}^{2}(I)$ can be expanded as follows:

$$
y(x)=\sum_{k=0}^{\infty} y_{k} R_{k}(x),
$$

with

$$
y_{k}=\frac{2}{\pi}\left\langle y, R_{k}\right\rangle_{w}
$$


The $y_{k}$ 's are the expansion coefficients associated with the family $\left\{R_{k}(x)\right\}$. If the infinite series in (2.8) is truncated, then it can be written as

$$
y(x) \simeq y_{N}(x)=\sum_{k=0}^{N} y_{k} R_{k}(x)=Y^{T} R(x),
$$

where $Y=\left[y_{0}, y_{1}, \ldots, y_{N}\right]^{T}$ and $R(x)=\left[R_{0}(x), R_{1}(x), \ldots, R_{N}(x)\right]^{T}$.

We can also approximate the function $k(x, t)$ in $L_{w}^{2}(I \times I)$ as follows:

$$
k(x, t) \simeq k_{N}(x, t)=R^{T}(x) K R(t),
$$

where $K$ is an $(N+1) \times(N+1)$ matrix that

$$
K_{i j}=\frac{2}{\pi^{2}}\left\langle R_{i}(x),\left\langle k(x, t), R_{j}(t)\right\rangle\right\rangle, \quad i, j=0,1, \ldots, N
$$

Moreover, from recurrence relation in (2.3) we have

$$
R(0)=\left[1,-2,3,-4, \ldots,(-1)^{N}(N+1)\right]^{T}=\mathbf{e}_{1} .
$$

\subsection{Product Integration of the RSC Functions}

We also use the matrix $P_{a b}$ as follows:

$$
P_{a b}=\int_{a}^{b} R(t) R^{T}(t) d t
$$

To illustrate the calculation $P_{a b}$ we choose $a=0$ and $b=1$, then we obtain

$$
P_{01}=\left[\begin{array}{cccccc}
1 & 2-4 \ln 2 & 11-16 \ln 2 & 28-40 \ln 2 & \frac{167}{3}-80 \ln 2 & \ldots \\
2-4 \ln 2 & 12-16 \ln 2 & 30-44 \ln 2 & \frac{200}{3}-96 \ln 2 & \frac{374}{3}-180 \ln 2 & \cdots \\
11-16 \ln 2 & 30-44 \ln 2 & \frac{203}{3}-96 \ln 2 & \frac{380}{3}-184 \ln 2 & \frac{3329}{15}-320 \ln 2 & \ldots \\
28-40 \ln 2 & \frac{200}{3}-96 \ln 2 & \frac{380}{3}-184 \ln 2 & \frac{3344}{15}-320 \ln 2 & \frac{5396}{15}-520 \ln 2 & \cdots \\
\frac{167}{3}-80 \ln 2 & \frac{374}{3}-180 \ln 2 & \frac{3329}{15}-320 \ln 2 & \frac{5396}{15}-520 \ln 2 & \frac{19451}{35}-800 \ln 2 & \cdots \\
\vdots & \vdots & \vdots & \vdots & \ddots
\end{array}\right] .
$$




\subsection{Operational Matrix of Derivative}

The derivative of the vector $R(x)$ defined in (2.10) can be approximated by

$$
R^{\prime}(x) \simeq D R(x),
$$

where $D$ is named the $n \times n$ operational matrix of derivative. Differentiating (2.3) we get

$$
\begin{gathered}
R_{0}^{\prime}(x)=0, \quad R_{1}^{\prime}(x)=\frac{5}{4} R_{0}(x)-R_{1}(x)+\frac{1}{4} R_{2}(x), \\
R_{n}^{\prime}(x)=\left(R_{1}(x) R_{n-1}(x)\right)^{\prime}-R_{n-2}^{\prime}(x), \quad n \geq 2 .
\end{gathered}
$$

By using (2.17) the matrix $D$ can be calculated. The matrix $D$ is a lower Hessenberg matrix and can be expressed as $D=D_{1}+D_{2}$, where $D_{1}$ is a tridiagonal matrix which is obtained from

$$
D_{1}=\operatorname{diag}\left(\frac{-2+7 i}{4},-i, \frac{i}{4}\right), \quad i=0, \ldots, n-1,
$$

and the $d_{i j}$ elements of matrix $D_{2}$ are obtained from

$$
d_{i j}= \begin{cases}0, & i \leq j+1 \\ (-1)^{i+j+1}(2 j), & i>j+1\end{cases}
$$

\subsection{The Product Operational Matrix}

The following property of the product of two rational Chebyshev vectors will also be used:

$$
R(x) R^{T}(x) Y=\tilde{Y} R(x)
$$

where $\tilde{Y}$ is called $(N+1) \times(N+1)$ product operational matrix for the vector $Y$. Using $(2.20)$ and the orthogonal property, the elements $\tilde{Y}_{i j}, i=0, \ldots, N, j=0, \ldots, N$ of the matrix $\tilde{Y}$ can be calculated from

$$
\tilde{Y}_{i j}=\frac{2}{\pi} \sum_{k=0}^{N} c_{k} g_{i j k}
$$

where $g_{i j k}$ is given by

$$
g_{i j k}=\int_{0}^{\infty} R_{i}(x) R_{j}(x) R_{k}(x) w(x) d x .
$$




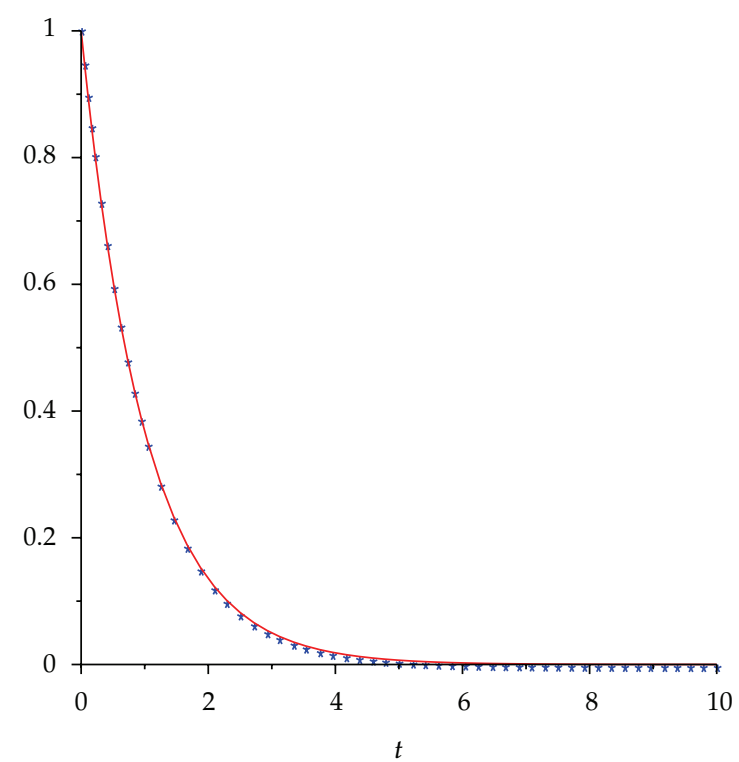

Figure 1: Graph of the exact and numerical solutions of $y_{1}(x)$ for $N=13$; symbols correspond to the numerical solution.

\section{Solving System of Integrodifferential Equations over Semi-Infinite Interval}

Consider the following system of integrodifferential equations:

$$
\begin{gathered}
\sum_{i=1}^{l}\left(\sum_{j=0}^{m} v_{p i j}(x) y_{i}^{(j)}(x)+\lambda_{i p} \int_{a}^{b}\left(k_{i p}(x, t) y_{i}(t)\right) d t\right)=f_{p}(x), \quad p=1,2, \ldots, l, \\
y_{i}^{(j)}(0)=y_{i j}, \quad i=1,2, \ldots, l, \quad j=0,1, \ldots, m-1, \\
x \in[0, \infty) .
\end{gathered}
$$

Using (2.10) and (2.11) to approximate $y_{i}, f_{p}, k_{i p}$, and $v_{p i j}$ when $i, p=1,2, \ldots, l$ and $j=$ $0, \ldots, m$, we have

$$
y_{i}(x) \simeq Y_{i}^{T} R(x), \quad f_{p}(x) \simeq F_{p}^{T} R(x), \quad k_{i p}(x, t) \simeq R^{T}(t) K_{i p} R(x), \quad v_{p i j}(x) \simeq V_{p i j}^{T} R(x)
$$

According to the operational matrix of derivative we can approximate $y_{i}^{(j)}$ as

$$
\begin{aligned}
y_{i}^{(j)}(x) & \simeq Y_{i}^{T} R^{(j)}(x) \simeq Y_{i}^{T} D^{j} R(x), \\
y_{i}^{(j)}(0) & \simeq Y_{i}^{T} D^{j} R(0)=Y_{i}^{T} D^{j} \mathbf{e}_{1} .
\end{aligned}
$$


With substituting these approximations in (3.1) we have

$$
\begin{gathered}
\sum_{i=1}^{l}\left(\sum_{j=0}^{m} Y_{i}^{T} D^{j} R(x) R^{T}(x) V_{p i j}+\lambda_{i p} \int_{a}^{b} Y_{i}^{T} R(t) R^{T}(t) K_{i p} R(x) d t\right)=F_{p}^{T} R(x), \\
Y_{i}^{T} D^{j} \mathbf{e}_{1}=y_{i j}, \quad i=1,2, \ldots, l, j=0,1, \ldots, m-1, p=1,2, \ldots, l .
\end{gathered}
$$

Then using (2.20) we obtain

$$
\begin{gathered}
\sum_{i=1}^{l}\left(\sum_{j=0}^{m} Y_{i}^{T} D^{j} \tilde{V}_{p i j} R(x)+\lambda_{i p} Y_{i}^{T}\left(\int_{a}^{b} R(t) R^{T}(t) d t\right) K_{i p} R(x)\right)=F_{p}^{T} R(x), \\
Y_{i}^{T} D^{j} \mathbf{e}_{1}=y_{i j}, \quad i=1,2, \ldots, l, j=0,1, \ldots, m-1, p=1,2, \ldots, l,
\end{gathered}
$$

which can be simplified using (2.14)

$$
\begin{gathered}
\sum_{i=1}^{l}\left(\sum_{j=0}^{m} Y_{i}^{T} D^{j} \tilde{V}_{p i j}+\lambda_{i p} Y_{i}^{T} P_{a b} K_{i p}\right)=F_{p}^{T}, \quad p=1,2, \ldots, l \\
Y_{i}^{T} D^{j} \mathbf{e}_{1}=y_{i j}, \quad i=1,2, \ldots, l, j=0,1, \ldots, n-1 .
\end{gathered}
$$

By solving this linear system of algebraic equations we can find vectors $Y_{i}, i=1,2, \ldots, n$, and then approximate the solutions $y_{i}(x)$ as

$$
y_{i}(x) \simeq Y_{i}^{T} R(x)
$$

\section{Numerical Examples}

Example 4.1. Consider the following system of linear integrodifferential equations:

$$
\begin{gathered}
\frac{1}{x+1} y_{1}^{\prime \prime}(x)+y_{1}^{\prime}(x)+y_{2}^{\prime \prime}(x)+\int_{0}^{1} \frac{y_{1}(t)+y_{2}(t)}{(x+1)^{2}(t+1)^{2}} d t=\frac{x-15}{4(x+1)^{3}} \\
y_{1}^{\prime}(x)-2 y_{2}^{\prime}(x)+y_{2}^{\prime \prime}(x)+24 \int_{0}^{1}\left(\frac{y_{1}(t)}{(x+1)^{2}(t+1)^{2}}+\frac{y_{2}(t)}{(x+1)^{3}(t+1)^{3}}\right) d t=\frac{8 x-1}{(x+1)^{3}} \\
y_{1}(0)=1, \quad y_{1}^{\prime}(0)=0, \quad y_{2}(0)=-1, \quad y_{2}^{\prime}(0)=2 .
\end{gathered}
$$

The exact solution of this example is $y_{1}(x)=1$ and $y_{2}(x)=(x-1) /(x+1)$. 
Table 1: Numerical results of Example 4.2.

\begin{tabular}{lcccccc}
\hline$x$ & \multicolumn{3}{c}{$y_{1}(x)$} & & & $y_{2}(x)$ \\
& $N=11$ & $N=13$ & Exact & $N=11$ & $N=13$ & Exact \\
\hline 0.0 & 1.00000 & 1.00000 & 1.00000 & 1.00000 & 1.00000 & 1.00000 \\
0.2 & 0.82435 & 0.81643 & 0.81873 & 0.67203 & 0.66964 & 0.67032 \\
0.4 & 0.67955 & 0.66654 & 0.67032 & 0.45203 & 0.44825 & 0.44933 \\
0.6 & 0.56024 & 0.54411 & 0.54881 & 0.30436 & 0.29994 & 0.30119 \\
0.8 & 0.46199 & 0.44410 & 0.44933 & 0.20516 & 0.20062 & 0.20190 \\
1.0 & 0.38119 & 0.36238 & 0.36788 & 0.13844 & 0.13413 & 0.13534 \\
1.2 & 0.31473 & 0.29559 & 0.30119 & 0.09351 & 0.08965 & 0.09072 \\
1.4 & 0.26007 & 0.24100 & 0.24660 & 0.06321 & 0.05991 & 0.06081 \\
1.6 & 0.21512 & 0.19637 & 0.20190 & 0.04274 & 0.04006 & 0.04076 \\
1.8 & 0.17819 & 0.15987 & 0.16530 & 0.02886 & 0.02681 & 0.02732 \\
2.0 & 0.14787 & 0.13003 & 0.13534 & 0.01942 & 0.01798 & 0.01832 \\
2.2 & 0.12300 & 0.10562 & 0.11080 & 0.01297 & 0.01211 & 0.01228 \\
2.4 & 0.10261 & 0.08565 & 0.09072 & 0.00855 & 0.00822 & 0.00823 \\
2.6 & 0.08589 & 0.06932 & 0.07427 & 0.00550 & 0.00565 & 0.00552 \\
2.8 & 0.07218 & 0.05596 & 0.06081 & 0.00339 & 0.00395 & 0.00370 \\
3.0 & 0.06094 & 0.04503 & 0.04970 & 0.00193 & 0.00285 & 0.00248 \\
\hline
\end{tabular}

We solved Example 4.1 using the present method with $N=3$, and we obtained $Y_{1}^{T}=$ $[1,0,0,0]$ and $Y_{2}^{T}=[0,0.5,0,0]$, which imply

$$
y_{1}(x)=Y_{1}^{T} R(x)=1, \quad y_{2}(x)=Y_{2}^{T} R(x)=\frac{x-1}{x+1}
$$

that are the exact solutions.

Example 4.2. Next, consider the following system of integrodifferential equations with the exact solution $y_{1}(x)=e^{-x}$ and $y_{2}(x)=e^{-2 x}$ :

$$
\begin{gathered}
y_{1}^{\prime \prime}(x)+y_{1}^{\prime}(x)+y_{2}^{\prime \prime}(x)+2 y_{2}^{\prime}(x)+\int_{0}^{1} 6 e^{-t-x}\left(y_{1}(t)+y_{2}(t)\right) d t=\left(5-3 e^{-2}-2 e^{-3}\right) e^{-x}, \\
4 e^{-x} y_{1}^{\prime \prime}(x)-y_{2}^{\prime \prime}(x)+\int_{0}^{1} 12 e^{-2(t+x)}\left(y_{1}(t)+y_{2}(t)\right) d t=\left(5-4 e^{-3}-3 e^{-4}\right) e^{-2 x}, \\
y_{1}(0)=1, \quad y_{1}^{\prime}(0)=-1, \quad y_{2}(0)=1, \quad y_{2}^{\prime}(0)=-2 .
\end{gathered}
$$

We solved this example by using the method described in Section 3 for $N=11$ and $N=13$. Results are shown in Table 1 and Figures 1 and 2. The errors for large values of $x$ are shown in Table 2. It is seen that the proposed method provides accurate results even for large values of $x$. 


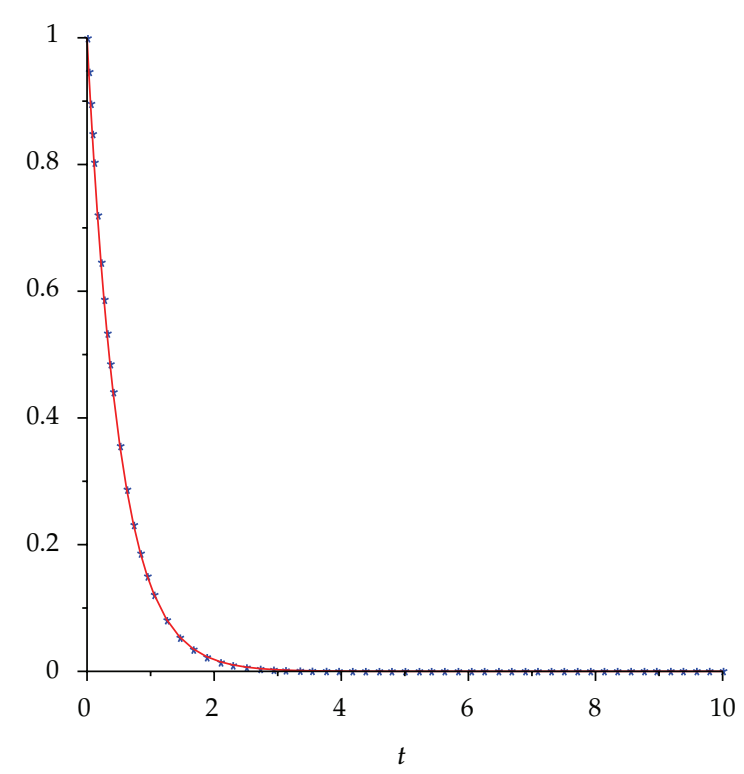

Figure 2: Graph of the exact and numerical solutions of $y_{2}(x)$ for $N=13$; symbols correspond to the numerical solution.

Table 2: Absolute error for large values of $x$.

\begin{tabular}{lcccc}
\hline & & & $N=11$ & \\
& $x=50$ & $x=100$ & $x=200$ & $x=400$ \\
\hline$e\left(y_{1}(x)-Y_{1}^{T} R(x)\right)$ & $3.1 \times 10^{-4}$ & $7.9 \times 10^{-5}$ & $1.1 \times 10^{-6}$ & $5.3 \times 10^{-7}$ \\
$e\left(y_{2}(x)-Y_{2}^{T} R(x)\right)$ & $4.5 \times 10^{-3}$ & $2.4 \times 10^{-3}$ & $1.2 \times 10^{-4}$ & $6.3 \times 10^{-5}$ \\
\hline
\end{tabular}

\section{Conclusion}

The fundamental goal of this paper has been to construct an approximation to the solution of the integrodifferential equations system in a semi-infinite interval. In the above discussion, the Galerkin method with RSC functions, which have the property of orthogonality, is employed to achieve this goal. Advantages of this method is that we do not reform the problem to a finite domain, and with a small value of $N$ accurate results are obtained. There is a good agreement between obtained results and exact values that demonstrates the validity of the present method for this type of problems and gives the method a wider applicability.

\section{References}

[1] J. Abdul Jerri, Introduction to Integral Equations with Applications, John Wiley \& Sons, New York, NY, USA, 1999.

[2] J. P. Boyd, "Evaluating of Dawson's integral by solving its differential equation using orthogonal rational Chebyshev functions," Applied Mathematics and Computation, vol. 204, no. 2, pp. 914-919, 2008.

[3] P. Linz, Analytical and Numerical Methods for Volterra Equations, vol. 7, Society for Industrial and Applied Mathematics, Philadelphia, Pa, USA, 1985.

[4] K. Maleknejad and Y. Mahmoudi, "Numerical solution of linear Fredholm integral equation by using hybrid Taylor and block-pulse functions," Applied Mathematics and Computation, vol. 149, no. 3, pp. 799-806, 2004. 
[5] Y. Ren, B. Zhang, and H. Qiao, "A simple Taylor-series expansion method for a class of second kind integral equations," Journal of Computational and Applied Mathematics, vol. 110, no. 1, pp. 15-24, 1999.

[6] M. T. Rashed, "Numerical solution of functional differential, integral and integro-differential equations," Applied Mathematics and Computation, vol. 156, no. 2, pp. 485-492, 2004.

[7] W. Wang, "An algorithm for solving the high-order nonlinear Volterra-Fredholm integro-differential equation with mechanization," Applied Mathematics and Computation, vol. 172, no. 1, pp. 1-23, 2006.

[8] S. Yalçınbaş and M. Sezer, "The approximate solution of high-order linear Volterra-Fredholm integrodifferential equations in terms of Taylor polynomials," Applied Mathematics and Computation, vol. 112, no. 2-3, pp. 291-308, 2000.

[9] S. Abbasbandy and A. Taati, "Numerical solution of the system of nonlinear Volterra integrodifferential equations with nonlinear differential part by the operational Tau method and error estimation," Journal of Computational and Applied Mathematics, vol. 231, no. 1, pp. 106-113, 2009.

[10] G. Ebadi, M. Y. Rahimi, and S. Shahmorad, "Numerical solution of the system of nonlinear Fredholm integro-differential equations by the operational tau method with an error estimation," Scientia Iranica, vol. 14, no. 6, pp. 546-554, 2007.

[11] A. Khani, M. Mohseni Moghadam, and S. Shahmorad, "Numerical solution of special class of systems of non-linear Volterra integro-differential equations by a simple high accuracy method," Bulletin of the Iranian Mathematical Society, vol. 34, no. 2, pp. 141-152, 2008.

[12] K. Maleknejad and M. Tavassoli Kajani, "Solving linear integro-differential equation system by Galerkin methods with hydrid functions," Applied Mathematics and Computation, vol. 159, no. 3, pp. 603-612, 2004.

[13] K. Maleknejad, F. Mirzaee, and S. Abbasbandy, "Solving linear integro-differential equations system by using rationalized Haar functions method," Applied Mathematics and Computation, vol. 155, no. 2, pp. 317-328, 2004.

[14] J. Biazar, H. Ghazvini, and M. Eslami, "He's homotopy perturbation method for systems of integrodifferential equations," Chaos, Solitons E Fractals, vol. 39, no. 3, pp. 1253-1258, 2009.

[15] E. Yusufoğlu, "An efficient algorithm for solving integro-differential equations system," Applied Mathematics and Computation, vol. 192, no. 1, pp. 51-55, 2007.

[16] A. Akyüz-Daşcioğlu and M. Sezer, "Chebyshev polynomial solutions of systems of higher-order linear Fredholm-Volterra integro-differential equations," Journal of the Franklin Institute, vol. 342, no. 6, pp. 688-701, 2005.

[17] B.-Y. Guo and J. Shen, "Laguerre-Galerkin method for nonlinear partial differential equations on a semi-infinite interval," Numerische Mathematik, vol. 86, no. 4, pp. 635-654, 2000.

[18] Y. Maday, B. Pernaud-Thomas, and H. Vandeven, "Shock-Fitting techniques for solving hyperbolic problems with spectral methods," Recherche Aerospatiale, vol. 6, pp. 1-9, 1985.

[19] H. I. Siyyam, "Laguerre tau methods for solving higher-order ordinary differential equations," Journal of Computational Analysis and Applications, vol. 3, no. 2, pp. 173-182, 2001.

[20] J. Shen, "Stable and efficient spectral methods in unbounded domains using Laguerre functions," SIAM Journal on Numerical Analysis, vol. 38, no. 4, pp. 1113-1133, 2000.

[21] J. P. Boyd, Chebyshev and Fourier Spectral Methods, Springer, Berlin, Germany, 2nd edition, 2000.

[22] B.-Y. Guo, "Jacobi spectral approximations to differential equations on the half line," Journal of Computational Mathematics, vol. 18, no. 1, pp. 95-112, 2000.

[23] J. P. Boyd, “Orthogonal rational functions on a semi-infinite interval," Journal of Computational Physics, vol. 70, no. 1, pp. 63-88, 1987.

[24] B.-Y. Guo, J. Shen, and Z.-Q. Wang, "A rational approximation and its applications to differential equations on the half line," Journal of Scientific Computing, vol. 15, no. 2, pp. 117-147, 2000.

[25] J. P. Boyd, C. Rangan, and P. H. Bucksbaum, "Pseudospectral methods on a semi-infinite interval with application to the hydrogen atom: a comparison of the mapped Fourier-sine method with Laguerre series and rational Chebyshev expansions," Journal of Computational Physics, vol. 188, no. 1, pp. 56-74, 2003.

[26] K. Parand and M. Razzaghi, "Rational Chebyshev tau method for solving higher-order ordinary differential equations," International Journal of Computer Mathematics, vol. 81, no. 1, pp. 73-80, 2004.

[27] H. I. Siyyam, "Laguerre Tau methods for solving higher-order ordinary differential equations," Journal of ComputationalAnalysis and Applications, vol. 3, no. 2, pp. 173-182, 2001.

[28] K. Parand and M. Razzaghi, "Rational Chebyshev tau method for solving Volterra's population model," Applied Mathematics and Computation, vol. 149, no. 3, pp. 893-900, 2004.

[29] K. Parand and M. Razzaghi, "Rational legendre approximation for solving some physical problems on semi-infinite intervals," Physica Scripta, vol. 69, no. 5, pp. 353-357, 2004. 
[30] M. Zarebnia and M. G. Ali Abadi, "Numerical solution of system of nonlinear second-order integrodifferential equations," Computers \& Mathematics with Applications, vol. 60, no. 3, pp. 591-601, 2010.

[31] M. Tavassoli Kajani and F. Ghasemi Tabatabaei, "Rational Chebyshev approximations for solving Lane-Emde equation of index $m$, " in Proceeding of the International Conference on Computational and Applied Mathematics, pp. 840-844, Bangkok, Thailand, March 2011.

[32] M. Abramowitz and I. A. Stegun, Handbook of Mathematical Functions, Dover, New York, NY, USA, 10th edition, 1972.

[33] M. Dadkhah Tirani, F. Ghasemi Tabatabaei, and M. Tavassoli Kajani, "Rational second (third) kind Chebyshev approximations for solving Volterras population model," in Proceeding of the International Conference on Computational and Applied Mathematics, pp. 835-839, Bangkok, Thailand, March 2011. 


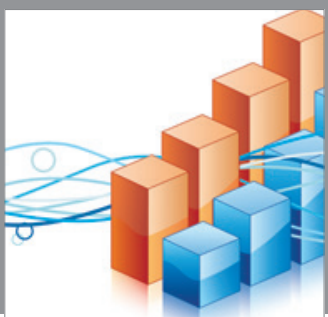

Advances in

Operations Research

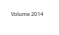

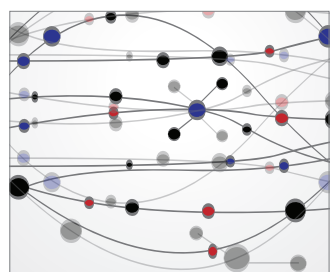

\section{The Scientific} World Journal
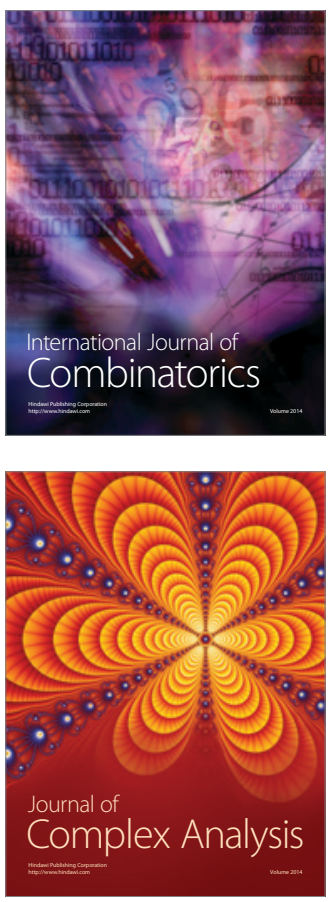

International Journal of

Mathematics and

Mathematical

Sciences
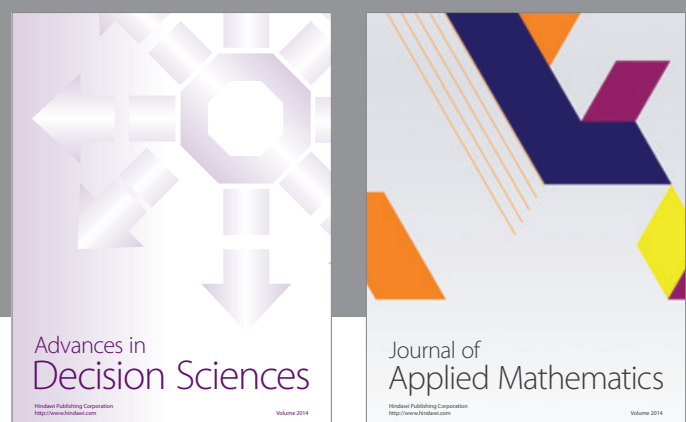

Journal of

Applied Mathematics
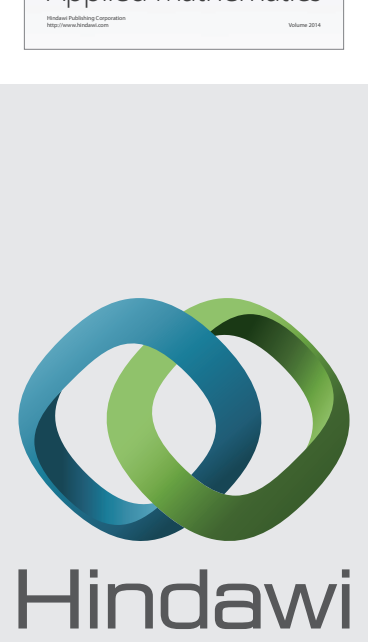

Submit your manuscripts at http://www.hindawi.com
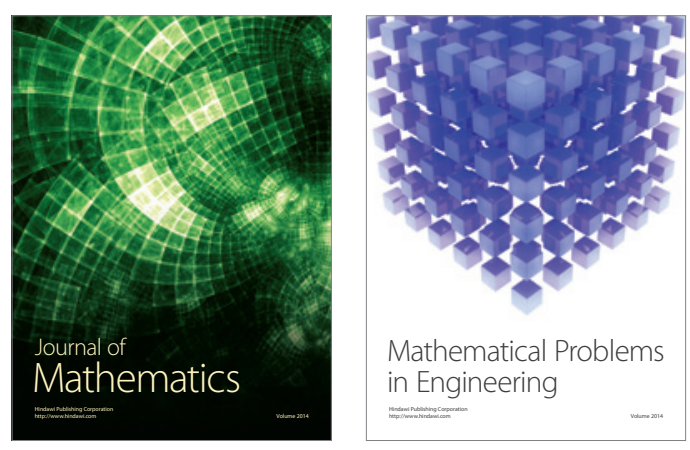

Mathematical Problems in Engineering
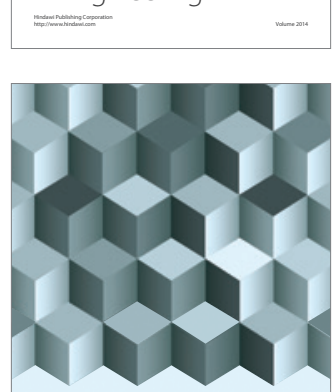

Journal of

Function Spaces
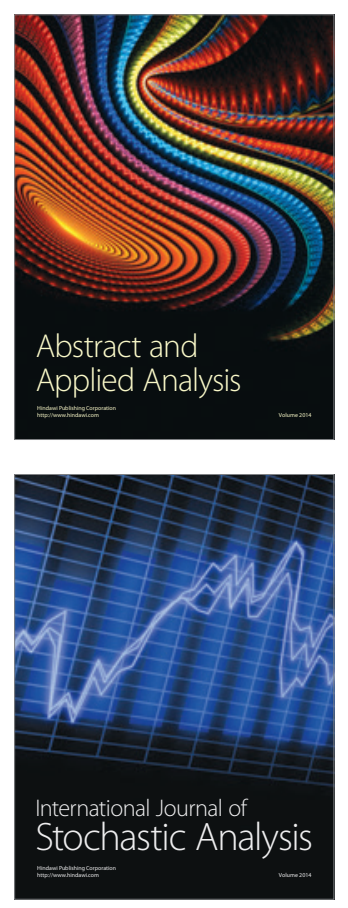

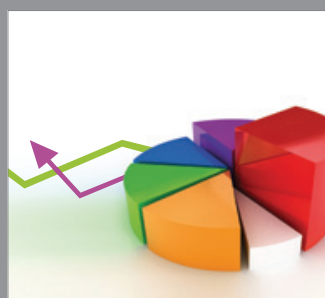

ournal of

Probability and Statistics

Promensencen
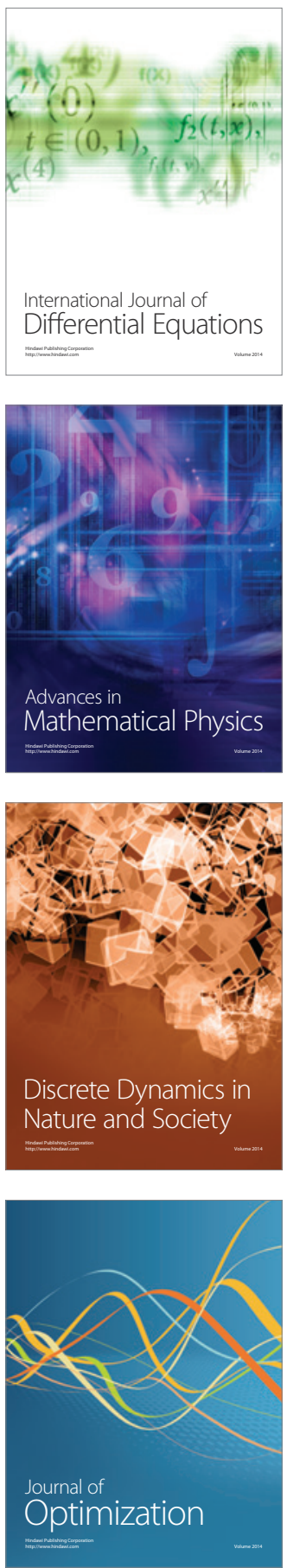